\title{
2966. A roller bearing fault diagnosis method using interval support vector deterministic optimization based on nested PSO
}

\author{
Qinge Dai ${ }^{1}$, Yongqi Chen ${ }^{2}$, Yang Chen ${ }^{3}$ \\ College of Science and Technology, Ningbo University, Ningbo, 315211, P. R. China \\ ${ }^{2}$ Corresponding author \\ E-mail: 1daiqinge@nbu.edu.cn, ${ }^{2}$ chenyongqi@nbu.edu.cn, ${ }^{3}$ chenyang@nbu.edu.cn \\ Received 6 January 2018; received in revised form 4 September 2018; accepted 15 October 2018 \\ DOI https://doi.org/10.21595/jve.2018.19613
}

Check for updates

Copyright $(2018$ Qinge Dai, et al. This is an open access article distributed under the Creative Commons Attribution License, which permits unrestricted use, distribution, and reproduction in any medium, provided the original work is properly cited.

\begin{abstract}
An interval support vector deterministic optimization model (ISVD) is proposed for the fault classification problem of uncertainty samples in this paper. Firstly, based on the order relation theory, support vector machines using interval samples is transformed into ISVD, which is a two-objective optimization problem, with the midpoint and the radius optimized at the same time. Then, ISVD is converted into a single objective optimization model by the linear combination. The single objective optimization model includes Lagrange multiplier vector and interval sample vectors, both of which are nested. Thus, the nested particle swarm optimization (PSO) based on dynamic decreasing inertia weight is applied to select the optimal Lagrange multiplier vector of this model. Lastly, the effectiveness of the proposed method is proved by the data set of University of California Irvine (UCI) and the roller bearing fault experiments. The experimental results show: ISVD owns outstanding generalization ability with the help of the structured risk minimization and global optimization. The accuracy of the proposed ISVD is better than that of the native Bayes uncertain classification method 1 (NBU1), native Bayes uncertain classification method 2 (NBU2) and the formula-based Bayes classifier (FBC).
\end{abstract}

Keywords: roller bearing, ISVD, nested PSO, interval.

\section{Introduction}

As a key component of the mechanical system, roller bearing often causes disorder for the race fault, ball fault etc. These faults bring great security risks to the mechanical system. Therefore, the fault diagnosis of roller bearing is of tremendous practical significance in engineer [1-4]. Traditional fault diagnosis methods only aim at deterministic fault samples, ignoring the uncertainty of the samples. However, the uncertainty of the samples should never be ignored. For these years, many scholars have harvested some representative research achievements in uncertainty interval data classification. Based on probability density, Sutar puts forward an interval data classification which extends classical decision tree algorithms to handle data with interval values [5]. Li et al. propose an interval data decision tree classification algorithm which combined with evidence theory [6]. Naive Bayes, as a widely used classification method for deterministic samples, has aroused a lot of interest among relevant researchers. Qin et al. create NBU1 and NBU2 [7]. NBU1 and NBU2 both use the parameter estimation method based on Gauss distribution assumption to calculate the class conditional probability density function of interval data. There is a slight difference between the above two methods in processing of the interval data model. However, NBU1 and NBU2 need the accuracy type of the class conditional probability density function of uncertain data. FBC algorithm, proposed by Ren etc., assumes uncertain interval data to meet the Gauss distribution [8]. The non parametric estimation in the Parzen window method is applied to calculate the types of the class conditional probability density function of interval samples. However, as a lazy learning method, FBC needs all training samples to predict each rolling bearing fault repeatedly. Therefore, both the computational complexity and the memory requirements of this method are too enormous to be offered in fault diagnosis fields. 
Support vector machines (SVM) is proposed by Vapnik [9]. Since structured risk minimization is applied in this method, it enables SVM to generalize better in the unseen interval testing samples than neural networks etc. which apply empirical risk minimization [10]. SVM can solve various problems in most of learning methods, such as small samples, nonlinearity, over fitting, high dimension. To make full use of these advantages, an ISVD model is proposed for the fault classification problem of uncertainty roller bearing samples. Meanwhile, the midpoint and the radius are optimized in this deterministic model. These two deterministic objective functions can be converted into a single objective optimization model by the linear combination. ISVD has Lagrange multiplier vector and interval sample vectors. These two groups of vectors are nested. The nested PSO algorithm is constructed based on the dynamic decreasing inertia weight PSO [11]. The advantages of nested PSO include excellent global optimization ability, fast convergence speed and easy implementation etc. Therefore, in this paper, the nested PSO is used to select the optimal decision variable and uncertain variable of ISVD.

The following parts of this paper is organized as the following. Section 2 reviews the order relation of interval number briefly. Support vector machines are introduced in Section 3, while an ISVD model is proposed in Section 4. Section 5 claims an optimization solver method based on the nested PSO for ISVD. Section 6 analyzes the classification results of UCI data and roller bearing faults, as well as the comparison results with other methods. Finally, the conclusions are presented in Section 7.

\section{The theory of order relation}

The order relation of interval number is proposed by Ishibuchi et al. [12]. For the minimization optimization problem, order relation $\leq_{m w}$ of interval number is defined as following:

$$
\begin{aligned}
& A \leq_{m w} B, \quad m(A) \geq m(B), \quad w(A) \geq w(B), \\
& A<_{m w} B, A \leq_{m w} B, \quad A \neq B, \\
& m(A)=\frac{A^{L}+A^{R}}{2}, \quad w(A)=\frac{A^{R}-A^{L}}{2}, \\
& m(B)=\frac{B^{L}+B^{R}}{2}, \quad w(B)=\frac{B^{R}-B^{L}}{2} .
\end{aligned}
$$

From Eq. (1), when an interval number $A$ is worse $\left(\leq_{m w}\right)$ than another interval number $B$, the midpoint $m(A)$ and the radius $w(A)$ are bigger than or equal to $m(B)$ and $w(B)$;

For maximum optimization problems, Eq. (1) is converted as follows:

$$
\begin{aligned}
& A \leq_{m w} B, \quad m(A) \leq m(B), \quad w(A) \leq w(B), \\
& A<_{m w} B, \quad A \leq_{m w} B, \quad A \neq B .
\end{aligned}
$$

Through using $\leq_{m w}$ to compare the objective function, one can know that the interval of the objective function caused by the uncertainty has not only a small midpoint but also a small radius. Thus, the uncertain objective function can be transformed into a two-objective optimization problem. One objective optimization problem is applied to get the smallest midpoint. The other optimization problem is utilized to obtain the smallest radius [13].

\section{The theory of SVM}

SVM is a machine learning method which is based on the structured risk minimization. In SVM, the classification can not only separate the two types of samples correctly, but also make the maximum classification distance. The former ensures the minimum of the empirical risk, and the latter is actually to minimize the confidence range. Let $\left\{\left(\mathbf{x}_{1}, y_{1}\right), \ldots,\left(\mathbf{x}_{n}, y_{n}\right)\right\} \subset \chi \times R$ be training data. The objective function of SVM can be described as following: 
$\min _{\omega, b, \xi_{i}} J=\frac{1}{2} w^{T} w+\frac{1}{2} c \sum_{j=1}^{n} \xi_{j}^{2}$,

s.t. $\left.y_{j}\left(w^{T} \mathbf{x}_{j}\right)+b\right) \geq 1-\xi_{j}$,

$\xi_{j}>0, j=1, \ldots, n$,

where $\xi_{j}$ is the slack variable, $c$ is the penalty parameter. This optimization problem, including the constraints, can be solved by the Lagrange function as follows:

$L(C, \beta, \mu, \xi, \alpha)=\frac{1}{2} w^{T} w+\frac{1}{2} c \sum_{j=1}^{n} \xi_{j}^{2}-\sum_{i=1}^{n} \alpha_{i}\left[y_{j}\left(w^{T} \mathbf{x}_{j}+b\right)-1-\xi_{j}\right]$.

Computing the partial derivatives, the solution of primal (4) can be calculated by working out the following Wolfe dual problem:

$\max \sum_{i=1}^{n} a_{i}-\frac{1}{2} \sum_{i=1}^{n} \sum_{j=1}^{n} \alpha_{i} \alpha_{j} y_{i} y_{j}\left(\mathbf{x}_{j} \mathbf{x}_{i}\right)$

s.t. $0 \leq a_{i} \leq C$,

$\sum_{i=1}^{n} a_{i} y_{i}=0, \quad i=1, \ldots, n$,

where $a_{i}$ is Lagrange multiplier. Only those points with $0 \leq a_{i} \leq C$ are named support vectors.

\section{The proposed ISVD optimization model}

Through the above treatments, the SVM optimization problem is a quadric program. But when the training sample is an uncertain interval number $\mathbf{x}_{i}=\left[x_{i}^{L}, x_{i}^{R}\right], \mathrm{SVM}$ is converted to ISVD model as follows:

$\min f(\mathbf{a}, \mathbf{x})=\frac{1}{2} \sum_{i=1}^{n} \sum_{j=1}^{n} \alpha_{i} \alpha_{j} y_{i} y_{j}\left(\left[x_{i}^{L}, x_{i}^{R}\right],\left[x_{j}^{L}, x_{j}^{R}\right]\right)-\sum_{i=1}^{n} a_{i}$,

s.t. $0 \leq a_{i} \leq C$,

$\sum_{i=1}^{n} a_{i} y_{i}=0, \quad i=1, \ldots, n$,

where $\mathbf{a}$ is the Lagrange multiplier vector, $\mathbf{x}$ is the uncertain interval samples vector. Through the above order relation $\leq_{m w}$ in Eq. (1), the optimal interval of the Eq. (6) has a small midpoint and a small radius simultaneously. For this reason, interval support vector optimization model in Eq. (6) can be converted ISVD optimization model. A deterministic two-objective programming problem is presented as:

$\min [m(f(\mathbf{a}, \mathbf{x}), w(f(\mathbf{a}, \mathbf{x}))]$,

s.t. $g(\mathbf{a})=\sum_{i=1}^{n} a_{i} y_{i}=0$,

$0 \leq a_{i} \leq C$, 


$$
\begin{aligned}
& m(f(\mathbf{a}, \mathbf{x}))=\frac{\min _{\mathbf{x} \in \Gamma} f(\mathbf{a}, \mathbf{x})+\max _{\mathbf{x} \in \Gamma} f(\mathbf{a}, \mathbf{x})}{2}, \\
& w(f(\mathbf{a}, \mathbf{x}))=\frac{\max _{\mathbf{x} \in \Gamma} f(\mathbf{a}, \mathbf{x})-\min _{\mathbf{x} \in \Gamma} f(\mathbf{a}, \mathbf{x})}{2},
\end{aligned}
$$

$\mathbf{a} \in \Omega^{n}, \mathbf{x} \in \Gamma=\left\{\mathbf{x} \mid \mathbf{x}^{L} \leq \mathbf{x} \leq \mathbf{x}^{R}\right\}, i=1, \ldots, n$.

This two objective programming problem includes the objective function and the constraints. A linear combination method is often used to dispose of the objective function [14]. The penalty function method is used to deal with the constraints [15]. Thus Eq. (7) can be converted into a single objective optimization model with a penalty function:

$$
\begin{aligned}
& \operatorname{minh}(\mathbf{a}, \mathbf{x})=\frac{\gamma w(f(\mathbf{a}, \mathbf{x}))}{\psi}+\frac{(1-\gamma) m(f(\mathbf{a}, \mathbf{x}))}{\varphi}+\sigma g(\mathbf{a}), \\
& \varphi=\min _{\mathbf{a} \in \Omega^{n}} m(f(\mathbf{a}, \mathbf{x})) \\
& \psi=\min _{\mathbf{a} \in \Omega^{n}} w(f(\mathbf{a}, \mathbf{x}))
\end{aligned}
$$

where $0 \leq \gamma \leq 1$ is a weighting factor of $m(f(\mathbf{a}, \mathbf{x}))$ and $w(f(\mathbf{a}, \mathbf{x})) . \varphi$ and $\psi$ are normalization factors, $\sigma$ is the penalty factor which is often set as a large value. Eq. (8) is a nested nonlinear optimization problem. For a Lagrange multiplier vector $\mathbf{a}$, the inner optimization processes are involved to obtain the smallest midpoint and radius for all the uncertain interval samples vector $\mathbf{x}$.

\section{Nested PSO based on dynamic decreasing inertia weight for ISVD model}

PSO algorithm, different from the genetic algorithm etc., is evolved by the cooperation and competition among individuals but not genetic operators. In the stage of evolution, PSO has many advantages, especially its excellent global optimization ability, simple operation and easy implementation, no selection, crossover, mutation and so on. In order to further improve the global optimization ability of PSO. This paper introduces a modified PSO using dynamically decreasing inertia weight [11]. As mentioned by Shi and Eberhart, when the inertia weight is low $(\omega \leq 0.8)$, the search time is very short, that is, all the particles tend to gather together quickly. This confirms that the PSO is more like a local search algorithm when $\omega$ is small. If there is an acceptable solution within the initial search space, then the PSO will find the global optimum quickly, otherwise it will not find the global optimum. On the contrary, when the inertia weight is large $(\omega \geq 1.2)$, the PSO algorithm always explores new regions. Of course, the PSO algorithm needs more iteration to reach the global optimal, and it is more likely not to find the global optimal. When the inertia weight is moderate $(0.8<\omega<1.2)$, the PSO will have the best chance to find the global optimum but also takes a moderate number of iterations. According to these analyses, Shi and Eberhart do not set the inertia weight as the fixed value, but set it to a function that decreases linearly with time, and the function of the inertia weight is:

$\omega=\omega_{\max }-\frac{\omega_{\max }-\omega_{\min }}{i t e r_{\max }} \cdot k$.

The detailed algorithm of PSO based on dynamic decreasing inertia weight can be summarized as follows:

Step 1: Particles are set as the random initial position $x_{i}^{k}, i=1, \ldots, n_{s w}$ and the random initial velocity $v_{i}^{k}, i=1, \ldots, n_{s w}$ in the search space.

Step 2: The fitness value is calculated for each particle.

Step 3: Compares each particle with its fitness $f_{i d}^{k}$ and individual extreme $p b e s t_{i d}^{k}$. If $f_{i d}^{k}>$ pbest $t_{i d}^{k}$, pbest $t_{i d}^{k}$ is replaced by $f_{i d}^{k}$. 
Step 4: updates the velocity and position of each particle according to the following equations:

$v_{i d}^{k+1}=\omega \cdot v_{i d}^{k}+c 1 \cdot \operatorname{rand}() \cdot\left(\right.$ pbest $\left._{i d}^{k}-x_{i d}^{k}\right)+c 2 \cdot \operatorname{rand}() \cdot\left(g b e s t_{i d}^{k}-x_{i d}^{k}\right)$,

$x_{i d}^{k+1}=x_{i d}^{k}+v_{i d}^{k+1}$,

$\omega=\omega_{\max }-\frac{\omega_{\max }-\omega_{\min }}{\text { iter }_{\max }} \cdot k$.

Step 5: If the end condition is satisfied, the algorithm is exit. Otherwise, $k=k+1$ and go back to Step 2 .

For these advantages of dynamic decreasing inertia weight PSO, a nested PSO is proposed to select the optimal Lagrange multiplier vector a of Eq. (8). There are two level dynamic decreasing inertia weight PSO in the nested PSO. The outer dynamic decreasing inertia weight PSO (outer PSO) is used to obtain the optimal value of the objective function $\operatorname{minh}(\mathbf{a}, \mathbf{x})$. The deterministic Lagrange multiplier vector $\mathbf{a}$ is used as the particle in this PSO. The inner dynamic decreasing inertia weight PSO (inner PSO) is applied to get the $\min _{\mathbf{x} \in \Gamma}(f(\mathbf{a}, \mathbf{x}))$ and $\max _{\mathbf{x} \in \Gamma}(f(\mathbf{a}, \mathbf{x}))$. The uncertain training sample vector $\mathbf{x}$ is used as the particle in inner PSO. For computing each interval of the objective function $f(\mathbf{a}, \mathbf{x})$, the outer PSO will call for two cycles of the inner PSO. Then the midpoint value $m(f(\mathbf{a}, \mathbf{x}))$ and radius of the interval function $w(f(\mathbf{a}, \mathbf{x}))$ are calculated. The maximum generations are employed as stopping criterion for nested PSO. This paper draws the flow chart of the nested PSO for interval support vector deterministic optimization model in Fig. 1.

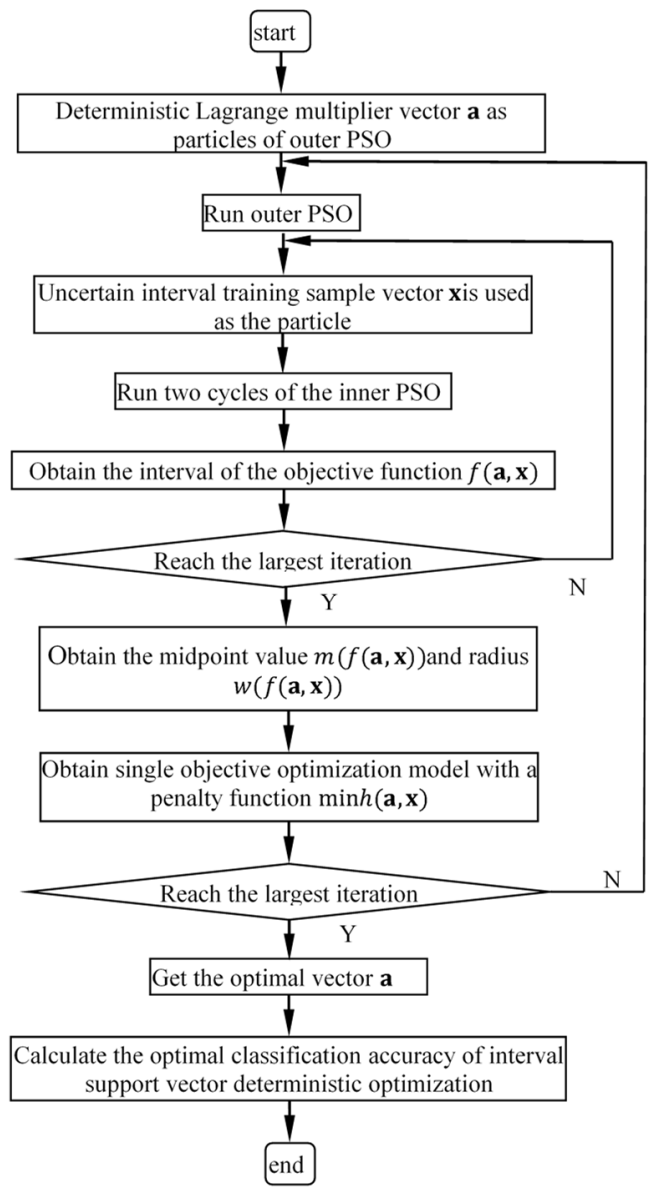

Fig. 1. Flow chart of nested PSO for ISVD model 


\section{The experiment}

To check the validity of the proposed ISVD, an UCI data set and the roller bearing fault experiments are carried out in this section.

\subsection{The classification of UCI data}

In this experiment, UCI iris data [16] set are used to test the effectiveness of ISVD based on nested PSO. Since there is not a set of standard uncertain data set in UCI, this experiment introduces uncertain information on the UCI data sets. The detailed method for adding uncertain information is as follows [17]:

For any UCI samples $\mathbf{x}_{i}=\left(x_{i 1}, x_{i 2}, \ldots, x_{i m}\right), i=1, \ldots, n$, one can add the interval uncertain information to each feature vector. The interval feature vector is listed as follows:

$\left[\underline{x}_{i j}, \bar{x}_{i j}\right]=\left[x_{i j}-r_{j}, x_{i j}+r_{j}\right], \quad i=1, \ldots, n, \quad j=1,2, \ldots, m$,

where $r_{j}$ is the interval radius. It can be obtained is as follows:

$r_{j}=\lambda \frac{\max \left(x_{i j}\right)-\min \left(x_{i j}\right)}{20}, \quad j=1,2, \ldots, m$.

The difference between $\max \left(x_{i j}\right)$ and $\min \left(x_{i j}\right)$ indicates the range of the sample set on the dimension $j . \lambda$ is a parameter that controls the size of the interval noise. For example: if $\lambda=1$, the distribution range of each attribute is added $10 \%$ noise. The interval samples of Iris are shown in Table 1.

Table 1. interval samples of iris

\begin{tabular}{|c|c|c|c|c|}
\hline Iris type & Sepal length & Sepal width & Petal length & Petal width \\
\hline \multirow{2}{*}{ Setosa } & {$[5.05,5.15]$} & {$[3.45,3.55]$} & {$[1.38,1.42]$} & {$[0.185,0.215]$} \\
\cline { 2 - 6 } & {$[4.85,4.95]$} & {$[2.95,3.05]$} & {$[1.38,1.42]$} & {$[0.185,0.215]$} \\
\hline \multirow{2}{*}{ Versicolour } & {$[6.395,6.605]$} & {$[2.75,2.85]$} & {$[4.52,4.68]$} & {$[1.47,1.53]$} \\
\cline { 2 - 6 } & {$[5.595,5.805]$} & {$[2.75,2.85]$} & {$[4.42,4.58]$} & {$[1.27,1.33]$} \\
\hline \multirow{2}{*}{ Virginica } & {$[6.565,6.835]$} & {$[2.445,2.555]$} & {$[5.695,5.905]$} & {$[1.76,1.84]$} \\
\cline { 2 - 6 } & {$[7.065,7.335]$} & {$[3.545,3.655]$} & {$[5.995,6.205]$} & {$[2.46,2.54]$} \\
\hline
\end{tabular}

In order to ensure the stability, this paper uses $90 \%$ of cross validation (10-fold Cross validation) estimation. The data sets are randomly divided into 10 groups. One group is taken turns to be chosen as the test set and the other 9 groups as the training set. Averaging 10 times is the result of the final classification accuracy. By using the training set, an interval support vector deterministic optimization is constructed in Eq. (8). In this equation, $\mathbf{x}$ is the interval training samples. $\gamma$ is a weighting factor. The larger $\gamma$ means that the preference of the radius of objection function is increased, while the preference of the midpoint is reduced. In this experiment, $\gamma$ is set 0.5 which means that the radius and midpoint are given a same preference. The proposed interval support vector deterministic optimization based on nested PSO has been implemented in MatLab 2012. The computer owns $3.2 \mathrm{G} \mathrm{GHz}$ Core (TM)2 i3 CPU and $2.0 \mathrm{G}$ memory. The operation system is Microsoft Windows 7. The detailed information of nested PSO is as follows:

In outer PSO:

(1) Lagrange multiplier vector a as particle of outer PSO. Number of particles: $n=25$;

(2) The largest iteration number: iter $_{\max }=100$;

(3) Inertia weight of PSO: $\omega_{\max }=1.2, \omega_{\min }=0.7298$;

(4) Positive acceleration constants: $c_{1}=1.4962, c_{2}=1.4962$;

In inner PSO, uncertain interval training sample vector $\mathbf{x}$ is used as the particle of inner PSO. The other parameters are the same as the outer PSO. Iris data has three types, but ISVD only aims 
to tackle two classification problems. For this reason, classification problems of iris data are divided into three groups classification problems. The detailed classification types are: Setosa vs Versicolour, Setosa vs Virginica, Versicolour vs Virginica. The nested PSO runs three times to solve these two classification problems. The convergence result of nested PSO is shown in Fig. 2 and Table 2.

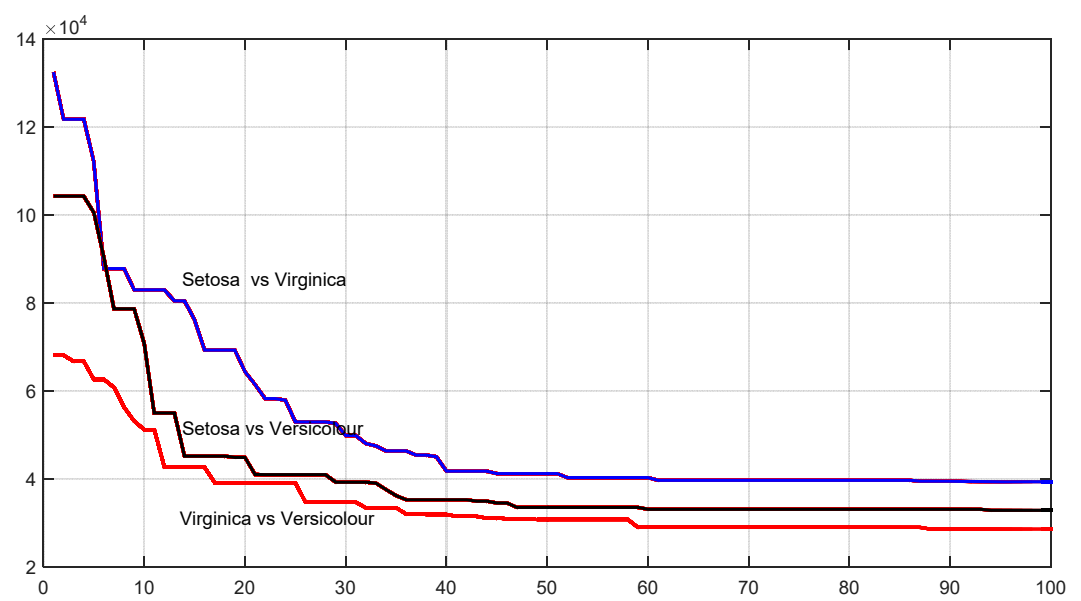

Fig. 2. Convergence performance of nested PSO in ISVD

Table 2. Optimization results for each two classification problem

\begin{tabular}{|c|c|c|c|c|}
\hline- & $\begin{array}{c}\text { The interval object } \\
\text { function }\end{array}$ & $\begin{array}{c}\text { The midpoint of } \\
\text { object function }\end{array}$ & $\begin{array}{c}\text { The radius of } \\
\text { object function }\end{array}$ & $\begin{array}{c}\text { The value of } \\
\text { penalty function }\end{array}$ \\
\hline $\begin{array}{c}\text { Setosa vs } \\
\text { Versicolour }\end{array}$ & {$\left[5.3715 \times 10^{4}, 7.4131 \times 10^{4}\right]$} & $6.3923 \times 10^{4}$ & $1.0208 \times 10^{4}$ & 2.0125 \\
\hline $\begin{array}{c}\text { Setosa vs } \\
\text { Virginica }\end{array}$ & {$\left[6.2817 \times 10^{4}, 9.8769 \times 10^{4}\right]$} & $8.0793 \times 10^{4}$ & $1.7976 \times 10^{4}$ & 1.0314 \\
\hline $\begin{array}{c}\text { Versicolour vs } \\
\text { Virginica }\end{array}$ & {$\left[2.8714 \times 10^{4}, 4.9730 \times 10^{4}\right]$} & $3.9222 \times 10^{4}$ & $1.0508 \times 10^{4}$ & 0.9857 \\
\hline
\end{tabular}

From Fig. 2 and Table 2, it can be found that the iterative solutions of nested PSO can approach the optimum in the 87 iterations. Especially for Setosa vs Virginica, only in 61 iterations, the value of the single objective optimization model combined with midpoint and radius is reduced from $1.265 \times 10^{5}$ to $4.13 \times 10^{4}$. Hence, the nested PSO has a higher convergence velocity. Through nested PSO, the optimal Lagrange multiplier vector a can be obtained and the optimal classification accuracy of ISVD can be calculated finally. The classification accuracy to analyze the performance of the proposed ISVD, NBU1, NBU2 and FBC is demonstrated in Table 3.

Table 3. Classification accuracy comparison for Iris

\begin{tabular}{|c|c|c|c|c|}
\hline Iris types & NBU1 & NBU1 & FBC & The proposed ISVD \\
\hline Setosa & $100 \%$ & $100 \%$ & $100 \%$ & $100 \%$ \\
\hline Versicolour & $95 \%$ & $95 \%$ & $95 \%$ & $95 \%$ \\
\hline Virginica & $92.5 \%$ & $92.5 \%$ & $95 \%$ & $97.5 \%$ \\
\hline
\end{tabular}

According to Table 3, the accuracy of the proposed method is $100 \%, 95 \%, 97.5 \%$ for Iris Setosa, Iris Versicolour and Iris Virginica respectively. The classification accuracy of the proposed method is $5 \%$ higher than that of NBU1 and NBU2 in the Iris Virginica type. For all the three Iris types, the accuracy of the proposed method is $97.5 \%$. The accuracy of NBU1 and NBU2 is only $95.83 \%$. Classification accuracy of NBU1 and NBU2 algorithm is the same, which is reasonable since the NBU1 and NBU2 are similar and these two methods are only different in the 
equation of average value and standard deviation. The accuracy of FBC is $96.67 \%$. However, as a lazy learning method, FBC needs all training samples to predict each rolling bearing fault repeatedly. Therefore, the memory requirements of this method are too enormous to be offered in fault diagnosis fields. The accuracy of the ISVD is higher than that of NBU1, NBU2 and FBC because the proposed method is constructed based on structured risk minimization theory. This theory pays attention to the empirical risk minimization and the model complexity minimization simultaneously. For this reason, the over fitting problem can be avoided and the proposed method has higher generalization ability for testing samples. Furthermore, based on dynamic decreasing inertia weight, nested PSO can obtain the optimal Lagrange multiplier vector a and improves the accuracy of ISVD because of its excellent global optimization ability

\subsection{Fault diagnosis for the roller bearing}

To verify the effectiveness of the proposed method, experiments are carried out on a roller bearing fault test platform in Fig. 3. In this test platform of Case Western Reserve University, an AC motor (2 hp) drives a shaft as the moving output. The corresponding speed of this motor can reach $1730 \mathrm{rpm}$. The test roller bearing supports the motor shaft at the drive end. The deep groove ball bearing 6205-2RS JEM SKF is selected in the tests platform. Bearing state consists of four categories: normal, inner race fault, outer race fault and ball fault.

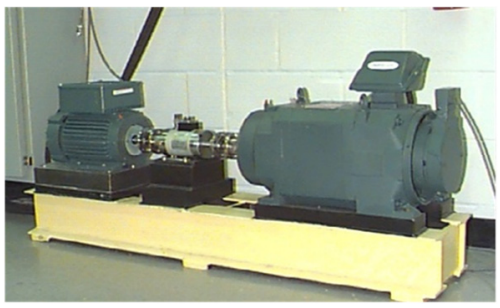

a)

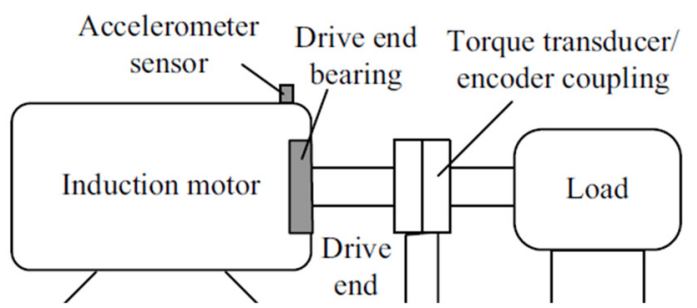

b)

Fig. 3. a) Motor drive fault test platform, b) schematic diagram of the tests platform

Status of fault damage bearings is a single damage. Damage diameter is divided into 7 mils, 14 mils, 21 mils and 28 mils. The accelerometers are used to collect vibration data. These data can be stored by a recorder at a sampling frequency of $12 \mathrm{kHz}$ for different bearing conditions.

Various defect roller bearing is produced by the electro-discharge machining in this test platform. Different roller bearings, such as inner race fault, outer race fault and ball fault are used to examine the proposed approach. Some defect roller bearing is shown in Fig. 4.

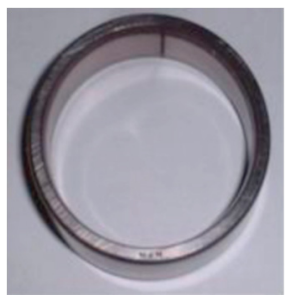

a)

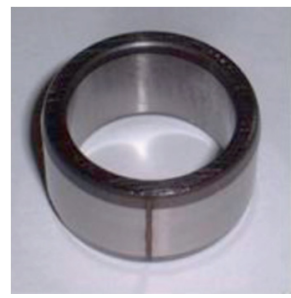

b)

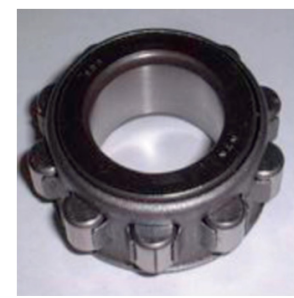

c)

Fig. 4. a) outer race fault, b) inner race fault, c) ball fault

Twelve fault types, such as normal bearing, inner race faults whose fault severity are 7 mils, 14 mils, 21 mils and 28 mils, outer race faults whose fault severity are 7 mils, 14 mils, 21 mils, ball faults whose fault severity are 7 mils, 14 mils, 21 mils and 28 mils, are used in this experiment. 


\subsubsection{Uncertain interval feature vectors acquisition}

In this section, each group of bearing fault vibration signal composed of 2048 points, is decomposed by intrinsic time scale decomposition (ITD) method in order to obtain a set of proper rotation components and the base component $[18,19]$. The first three groups of rotation component instantaneous amplitude and instantaneous frequency are applied, since they contain the main information. Then, the sample entropy of instantaneous amplitudes and instantaneous frequencies is calculated. Fault data of specific features extraction steps are shown as:

(1) Obtain the fault vibration data from the accelerometers of the fault test platform.

(2) Through ITD decomposition, the fault vibration data is decomposed of a set of rotation components and the baseline component. The decomposition results are given as Fig. 5.

(3) Obtain the first 3 group rotation components instantaneous amplitude, instantaneous frequency and instantaneous frequency.

(4) Calculate the sample entropy of the instantaneous amplitude and instantaneous frequency.

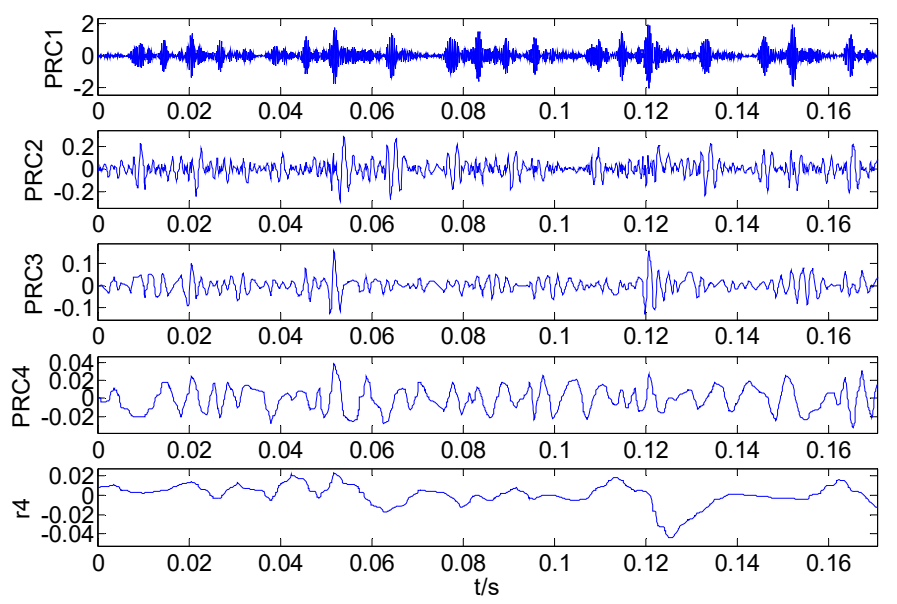

Fig. 5. Decomposition results gained by ITD for inner race fault (28 mils)

Each feature vector component is six-dimensional because it includes the sample entropy of the instantaneous phase and instantaneous frequency of the first 3 group rotation components. By this means, 100 samples for each kind of fault signal can be obtained. Since there is no open standard uncertainty rolling bearing fault number set, this experiment also introduces uncertainty information on the rolling bearing fault samples [13]. The detailed method for adding uncertainty information is the same as UCI iris mentioned above.

Then, a part of the training samples and testing samples are shown in Table 4 . The amplitude entropy 1 in Table 3 represents the first rotation component instantaneous amplitude sample entropy, other characteristics and so on.

\subsubsection{Comparative analysis of classification accuracy}

This section uses rolling bearing uncertain interval faults to compare the classification accuracy of NBU1, NBU2, FBC and the proposed method.

In order to ensure the stability of the test results, it is true that 10 -fold cross-validation method is also applied to verify the algorithm classification accuracy. Weighting factor of the proposed method $\gamma$ is set 0.5 . The experimental results are shown in Table 5. From Table 5, experimental results show that the classification accuracy of the proposed ISVD is better than NBU1, NBU2 and FBC. According to Inner race fault (14mils), accuracy of the proposed ISVD is $6.25 \%$ higher than FBC, NBU1 and NBU2. Moreover, the total accuracy can be calculated. The proposed ISVD: 
$97.08 \%$. The reason of such a high accuracy is structural risk minimization principle and the outstanding global optimization ability of nested PSO in the learning process of the proposed method. The accuracy of NBU1 is $94.79 \%$, while the accuracy of NBU2 is $94.58 \%$. Classification accuracy of NBU1 and NBU2 algorithm based on Naive Bias is very close, which is reasonable since these two methods are constructed based on Naive Bias and only different in the equation of average value and standard deviation. The accuracy of FBC: $95.31 \%$. However, the FBC algorithm is a kind of lazy learning method. It needs all training samples to predict each rolling bearing fault repeatedly. Both the computational complexity and memory requirements of this method are too large to be offered in roller bearing fault diagnosis.

Table 4. Fault samples for twelve kinds of bearing vibration signals

\begin{tabular}{|c|c|c|c|c|c|c|c|}
\hline $\begin{array}{c}\text { Fault } \\
\text { condition }\end{array}$ & $\begin{array}{c}\text { Fault } \\
\text { severity }\end{array}$ & $\begin{array}{c}\text { Amplitude } \\
\text { entropy 1 }\end{array}$ & $\begin{array}{c}\text { Amplitude } \\
\text { entropy 2 }\end{array}$ & $\begin{array}{c}\text { Amplitude } \\
\text { entropy 3 }\end{array}$ & $\begin{array}{c}\text { Frequency } \\
\text { entropy 1 }\end{array}$ & $\begin{array}{c}\text { Frequency } \\
\text { entropy 2 }\end{array}$ & $\begin{array}{c}\text { Frequency } \\
\text { entropy 3 }\end{array}$ \\
\hline Normal & 0 & {$[0.337,0.381]$} & {$[0.169,0.179]$} & {$[0.053,0.057]$} & {$[0.772,0.797]$} & {$[0.323,0.365]$} & {$[0.177,0.185]$} \\
\hline \multirow{4}{*}{$\begin{array}{c}\text { Inner race } \\
\text { fault }\end{array}$} & 7 & {$[0.745,0.756]$} & {$[0.423,0.429]$} & {$[0.171,0.175]$} & {$[0.960,0.967]$} & {$[1.058,1.070]$} & {$[0.589,0.599]$} \\
\cline { 2 - 8 } & 21 & {$[0.330,0.340]$} & {$[0.392,0.398]$} & {$[0.137,0.141]$} & {$[0.826,0.837]$} & {$[0.921,0.941]$} & {$[0.527,0.538]$} \\
\cline { 2 - 8 } & 28 & {$[0.340,0.347]$} & {$[0.302,0.309]$} & {$[0.093,0.099]$} & {$[0.527,0.534]$} & {$[0.676,0.687]$} & {$[0.463,0.472]$} \\
\hline \multirow{3}{*}{$\begin{array}{c}\text { Outer race } \\
\text { fault }\end{array}$} & 7 & {$[0.912,0.925]$} & {$[0.309,0.316]$} & {$[0.132,0.136]$} & {$[0.909,0.917]$} & {$[0.730,0.739]$} & {$[0.471,0.482]$} \\
\cline { 2 - 8 } & 14 & {$[0.993,1.010]$} & {$[0.359,0.372]$} & {$[0.113,0.118]$} & {$[0.841,0.852]$} & {$[0.823,0.847]$} & {$[0.438,0.449]$} \\
\hline \multirow{4}{*}{ Ball fault } & 21 & {$[0.138,0.143]$} & {$[0.225,0.234]$} & {$[0.073,0.077]$} & {$[0.909,0.918]$} & {$[0.674,0.683]$} & {$[0.416,0.428]$} \\
\cline { 2 - 8 } & 14 & {$[0.908,0.923]$} & {$[0.404,0.412]$} & {$[0.137,0.141]$} & {$[0.652,0.657]$} & {$[0.856,0.881]$} & {$[0.483,0.491]$} \\
\cline { 2 - 8 } & 21 & {$[0.822,0.778]$} & {$[0.390,0.405]$} & {$[0.118,0.124]$} & {$[0.809,0.829]$} & {$[0.878,0.921]$} & {$[0.489,0.504]$} \\
\cline { 2 - 8 } & 28 & {$[0.407,0.434]$} & {$[0.463,0.476]$} & {$[0.176,0.181]$} & {$[0.593,0.600]$} & {$[1.137,1.150]$} & {$[0.579,0.590]$} \\
\hline
\end{tabular}

Table 5. Classification accuracy comparison of four algorithms

\begin{tabular}{|c|c|c|c|c|c|}
\hline Fault condition & Target output & The proposed method & FBC & NBU1 & NBU2 \\
\hline Normal & 1 & $100 \%$ & $100 \%$ & $100 \%$ & $100 \%$ \\
\hline Ball (7mils) & 2 & $100 \%$ & $100 \%$ & $93.75 \%$ & $93.75 \%$ \\
\hline Inner race fault (7mils) & 3 & $100 \%$ & $100 \%$ & $100 \%$ & $100 \%$ \\
\hline Outer race fault (7mils) & 4 & $100 \%$ & $100 \%$ & $98.75 \%$ & $98.75 \%$ \\
\hline Ball (14mils) & 5 & $71.25 \%$ & $62.5 \%$ & $67.5 \%$ & $65 \%$ \\
\hline Inner race fault (14mils) & 6 & $100 \%$ & $93.75 \%$ & $93.75 \%$ & $93.75 \%$ \\
\hline Outer race fault (14mils) & 7 & $98.75 \%$ & $97.5 \%$ & $97.5 \%$ & $97.5 \%$ \\
\hline Ball (21mils) & 8 & $95 \%$ & $91.25 \%$ & $90 \%$ & $90 \%$ \\
\hline Inner race fault (21mils) & 9 & $100 \%$ & $100 \%$ & $100 \%$ & $100 \%$ \\
\hline Outer race fault (21mils) & 10 & $100 \%$ & $100 \%$ & $100 \%$ & $100 \%$ \\
\hline Ball (28mils) & 11 & $100 \%$ & $100 \%$ & $100 \%$ & $100 \%$ \\
\hline Inner race fault (28mils) & 12 & $100 \%$ & $98.75 \%$ & $96.25 \%$ & $96.25 \%$ \\
\hline
\end{tabular}

\section{Conclusions}

In this paper, ISVD is proposed by incorporating the interval number order relation theory into SVM, so that the advantages of SVM can be preserved. The main advantage is that the structural risk minimization principle enables the proposed method to generalize well in the unseen interval testing samples. Moreover, outstanding global optimization ability of nested PSO further improves the classification effect of the proposed method. Experiment results show that the proposed method has higher classification accuracy in roller bearing interval fault than NBU1, NBU2 and FBC. Nowadays, ISVD is still in the experimental verification stage based on the motor drive fault test platform. The proposed method will be used in the actual working environment. Its ability to resist noise needs further improvement in the near future, which will be the following important task of this research team 


\section{Acknowledgements}

This research is supported by Zhejiang Provincial Natural Science Foundation of China under Grant No. LY16E050001, Public Projects of Zhejiang Province (LGF18F030003), Ningbo Natural Science Foundation (2017A610138), sponsored by K.C. Wong Magna Fund in Ningbo University.

\section{References}

[1] Yang J. Y., Zhang Y. Y., Zhu Y. S. Intelligent fault diagnosis of rolling element bearing based on SVMs and fractal dimension. Mechanical Systems and Signal Processing, Vol. 21, 2007, p. 2012-2024.

[2] Shen C. Q., Wang D., Kong F. R. Fault diagnosis of rotating machinery based on the statistical parameters of wavelet packet paving and a generic support vector regressive classier. Measurement, Vol. 46, 2013, p. 1551-1564.

[3] Liu H. H., Han M. H. A fault diagnosis method based on local mean decomposition and multi-scale entropy for roller bearings. China Mechanical Engineering, Vol. 75, 2014, p. 64-68.

[4] Zhao Z. H., Yang S. P., Li S. H. Bearing fault diagnosis based on hilbert spectrum and singular value decomposition. Mechanical Systems and Signal Processing, Vol. 24, 2013, p. 346-350.

[5] Sutar R., Malunjkar A., Kadam A., et al. Decision trees for uncertain data. IEEE Transactions on Knowledge and Data Engineering, Vol. 23, 2011, p. 64-78.

[6] Li F., Li Y., Wang C. Uncertain data decision tree classification algorithm. Journal of Computer Applications, Vol. 29, Issue 11, 2009, p. 3092-3095.

[7] Qin B., Xia Y., Wang S., et al. A novel Bayesian classification for uncertain data. Knowledge-Based Systems, Vol. 24, Issue 8, 2011, p. 1151-1158.

[8] Ren J., Lee S. D., Chen X. L., et al. Naive Bayes classification of uncertain data. Proceedings of the 9th IEEE International Conference on Data Mining, Vol. 1, Issue 1, 2009, p. 944-949.

[9] Vapnik V. The Nature of Statistical Learning Theory. Springer, New York, 1995.

[10] Vapnik V. Statistical Learning Theory. Wiley, New York, 1998.

[11] Shi Y. H., Eberhart R. C. A modified particle swarm optimizer. IEEE International Conference on Evolutionary Computation, Vol. 4, 1998, p. 69-73.

[12] Ishibuchi H., Tanaka H. Multiobjective programming in optimization of the interval objective function. European Journal of Operational Research, Vol. 48, 1990, p. 219-225.

[13] Jiang C., Han X., Liu G. R., Liu G. P. A nonlinear interval number programming method for uncertain optimization problems. European Journal of Operational Research, Vol. 188, 2008, p. 1-13.

[14] Hu Y. D. Applied Multiobjective Optimization. Shanghai Science and Technology Press, 1990.

[15] Chen B. L. Optimization Theories and Algorithms. Tsinghua University Press, Beijing, 2002.

[16] Center for Machine Learning and Intelligent Systems, http://archive.ics.uci.edu/ml/datasets.html.

[17] Li W. J., Xiong X. F., Mao Y. M. Classification method for interval uncertain data based on improved naive Baye. Journal of Computer Applications, Vol. 11, Issue 10, 2014, p. 3268-3272.

[18] Frei M. G., Osorio I. Intrinsic time scale decomposition time-frequency-energy analysis and real-time filtering of non-stationary signals. Proceedings of Mathematical, Physical and Engineering Sciences, Vol. 463, Issue 10, 2007, p. 321-342.

[19] Feng Z. P., Lin X. F., Zuo M. J. Joint amplitude and frequency demodulation analysis based on intrinsic time-scale decomposition for planetary gearbox fault diagnosis. Mechanism and Machine Theory, Vol. 72, 2016, p. 223-240.

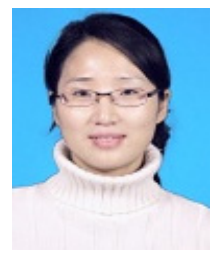

Qinge Dai received her Ph.D. degree from in Ningbo University, Ningbo, China, in 2013. Now she works in Ningbo University. Her research interests include fault diagnosis, intelligent algorithm. 


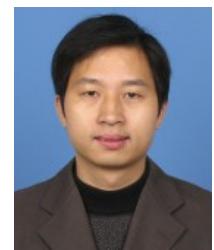

Yongqi Chen received his Ph.D. degree from in Tongji University, Shanghai, China, in 2012. Now he works in Ningbo University. His research interests include fault diagnosis, intelligent algorithm, statistical learning.

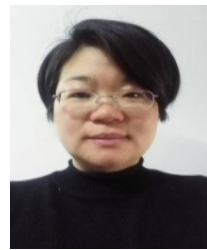

Yang Chen received her Ph.D. degree from in Zhejiang University, Hangzhou, China, in 2011. Now she works in Ningbo University. Her research interests include fault diagnosis, control algorithm. 